\title{
A Sudden Rise of Patients with Acute Macular Neuroretinopathy during the COVID-19 Pandemic
}

\author{
Maarten B. Jalink ${ }^{a, b}$ Inge H.G. Bronkhorst ${ }^{b}$ \\ aDepartment of Ophthalmology, University Medical Center Utrecht, Utrecht, \\ The Netherlands; ${ }^{b}$ Department of Ophthalmology, Jeroen Bosch Ziekenhuis, \\ 's-Hertogenbosch, The Netherlands
}

\section{Keywords}

COVID-19 - Vaccine $\cdot$ Acute macular neuroretinopathy

\begin{abstract}
The aim of this paper is to inform on the surge of cases of acute macular neuroretinopathy $(\mathrm{AMN})$ - a rare disease characterized by the sudden onset of acute scotomas caused by ischemia of the retinal capillary plexus - during the COVID-19 pandemic. In 2021, during the COVID-19 pandemic, a sudden rise in patients with AMN was observed in our clinic. In this paper, 4 cases from a hospital in the south of the Netherlands are reported, all of which could directly be linked to a COVID-19 infection or vaccination against the corona virus. A search for similar cases in the PubMed database produced thirteen relevant reports, which revealed that a similar increase in cases of AMN, all linked to COVID-19, has been observed worldwide. Analysis of the literature revealed that $A M N$ is seen more often during the pandemic and that AMN after COVID-19 happens at a significantly older age than typically reported. This is the largest case series of patients with AMN after COVID-19 infection or vaccination. With the ongoing pandemic and extensive vaccination programs, it is expected that cases of AMN will surge. It is important for ophthalmologists to be aware of this disease, especially since typical patient characteristics may differ.
\end{abstract}

\section{Karger"}




\section{Introduction}

Acute macular neuroretinopathy (AMN) is a rare, retinal, microvascular disorder that was first reported by Bos and Deutman in 1975 [1]. It is characterized by the acute onset of brown-red, wedge-shaped (or petaloid) perifoveal lesions that cause paracentral scotomas $[2,3]$. Symptoms arise acutely, can be unilateral or bilateral, and can either be temporary or permanent [4]. Usually, there is no progression or recurrence of the disease. The scotomas are relative and can be identified using perimetry or Amsler grid, and the typical lesions can be depicted with infra-red or red-free photography and optical coherence tomography (OCT) $[3,4]$. Fluorescein angiography is often normal, but might show minor abnormalities of the parafoveal capillaries $[1,4]$. Currently, the etiology of AMN is not completely understood. However, spectral-domain OCT studies have identified ischemia of the deep retinal capillary plexus as part of the disease's origin, seen as hyperreflectivity of the outer nuclear and plexiform layers [2, 3]. AMN is typically seen in females $(84.2 \%)$ of Caucasian heritage with an average age of 29.5 years and is correlated with (viral) flu-like illnesses, influenza vaccines, the use of oral contraceptives, and causes of vasospasm such as systemic shock or the use of vasoconstrictor drugs like epinephrine $[2,5,6]$. The prevalence of AMN is estimated at less than 1 patient per million [7], but this might be an underestimate since the disease is not well known and can be hard to diagnose. There are currently no treatment options, and it is unknown whether stopping oral contraceptives has influence on the course of the disease.

COVID-19, a primarily respiratory illness caused by the SARS-CoV-2 corona virus, is an entity that needs very little introduction at the time of writing $[8,9]$. The virus has rapidly spread and has led to millions of deaths worldwide, prompting governments to invoke drastic measurements such as curfews and lockdowns. Not all patients become symptomatic, and the degree of illness varies widely among patients. At least one-third of cases are estimated to have an asymptomatic course [10]. A wide variety of ophthalmic manifestations have been described [11]. In the past 2 years, over 10,000 scientific papers on the virus have been recorded in the PubMed database alone. Several vaccines have been developed and are deployed widely. In the Netherlands, $84.4 \%$ of adults were fully vaccinated at the time of writing [12].

During the first 11 months of 2021, a rise of patients with AMN was noted at the department of ophthalmology at the Jeroen Bosch Ziekenhuis in 's-Hertogenbosch, a general hospital in the south of the Netherlands. Before the COVID-19 pandemic, AMN was seen less than once a year. Now, however, 5 cases were seen, of which 4 could be linked to the pandemic. This paper discusses these 4 cases of AMN, followed by an analysis of similar cases in the recent literature.

\section{Case Series}

Patient A is a 42-year-old, healthy woman that had been using oral contraceptives for 10 years. She noticed a scotoma temporal to the center in her right eye, but only when closing her left one. She had received two vaccinations (Spikevax ${ }^{\circledR}$, COVID-19 mRNA vaccine; ModernaTX Inc., Cambridge, MA, USA), of which the second one was 1.5 months prior to the discovery of this scotoma. As far as known, the patient had not contracted COVID-19. Visual acuity and slit-lamp examination were normal. Fundoscopy of the right eye revealed a faint, brownish circle nasal to the fovea. OCT scan of the right eye showed subtle irregularities of the outer nuclear and photoreceptor layers at that exact same spot (Fig. 1). Humphrey Field Analyzer (HFA) 10-2 visual field test (Carl Zeiss AG, Jena, Germany) revealed a scotoma temporal to the center, as described by the patient. The patient was diagnosed with AMN and was advised to look for an alternative to her oral contraceptives. On 1-month follow-up, the scotoma was still present, but had diminished in size.

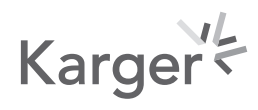


Fig. 1. OCT scan of the right eye of patient A; the infra-red image (up) shows a paracentral lesion (arrow); on the OCT image (down), only subtle changes to the outer nuclear and photoreceptor layers are seen.

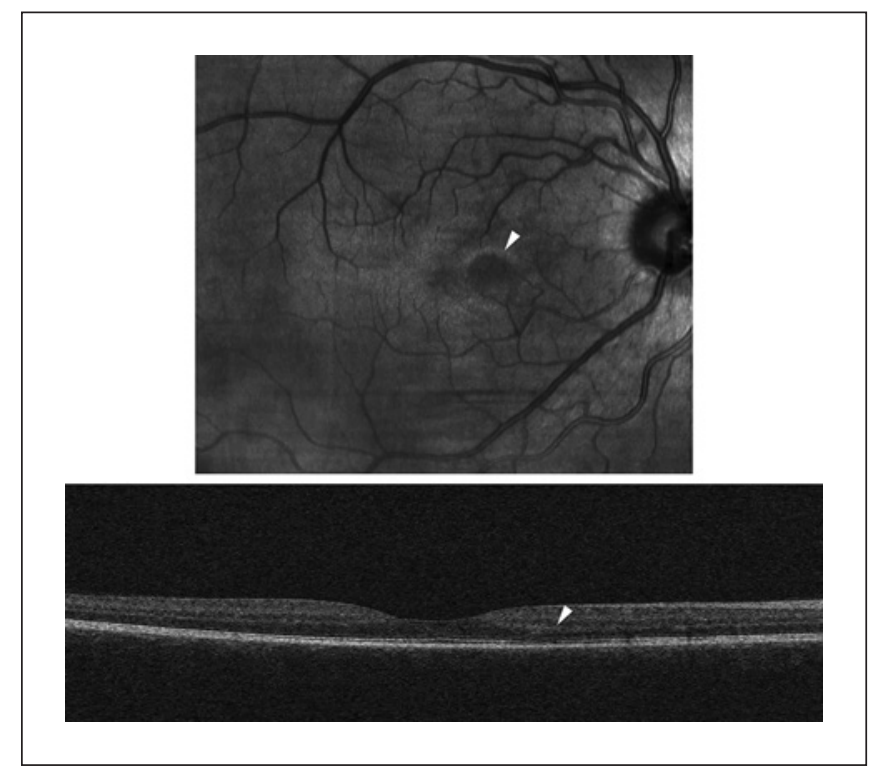

Patient B is a 29-year-old woman that presented with the sudden onset of a small, inferior temporal, transparent scotoma in her left eye since 2 weeks. The patient had switched from oral contraceptives to a hormonal intrauterine device 3 weeks before and had been tested positive for COVID-19 using a polymerase chain reaction test 5 months before. Interestingly, she had been referred 5 years earlier with a similar complaint that originated 5 months after experiencing a flu-like disease; at the time, nothing was noted on fundoscopy, and no OCT scan had been made. Visual acuity and slit-lamp examination were unremarkable. Fundoscopy of the left eye showed very faint alterations nasal superior to the fovea. Likewise, OCT scan only showed very subtle photoreceptor layer changes at that spot. The HFA 10-2 visual field test was normal. The scotoma remained unchanged over the course of 2 months. The patient was uneventfully vaccinated against COVID-19, 3 months after initial presentation.

Patient C is a healthy, 40-year-old woman that presented with blurry vision and photopsias in her right eye. She did not use any medication, had not had flu-like symptoms, and was never tested positive for COVID-19. The patient had her second COVID-19 vaccine (Spikevax; Moderna) 1.5 months prior to presentation. Visual acuity and slit-lamp examination were normal. On fundoscopy, mild pigmentary changes were noted nasal superior to the fovea. An HFA 10-2 visual field test showed a temporal inferior scotoma, corresponding to the findings on fundoscopy. The infra-red image of the OCT scan of this area showed a single round spot and neuroretinal changes comparable to the other 3 cases. Two months after presentation, her complaints were nearly gone, and OCT image and visual field test had returned to normal.

Patient D is a 21-year-old woman, unvaccinated against COVID-19 and only using oral contraceptives, who had COVID-19 6 weeks before presentation. She complained of a sudden scotoma temporal to the center in her right eye for 2 weeks and photopsias since 1 week. Visual acuity and slit-lamp examination were normal. Fundoscopy of the right eye revealed a round, brown spot nasal to the fovea, which was also visible on IR photography, but not on fundus photography. OCT scan of the right eye showed irregularities at the outer nuclear and photoreceptor layers (Fig. 2). The HFA 10-2 visual field test was normal. The left eye was normal on fundoscopy and OCT. The patient was advised to stop oral contraceptives. Followup (3 months) is still ongoing. 
Fig. 2. OCT scan of the right eye of patient D; the infra-red image (up) shows a small paracentral lesion (arrow), visible on the OCT image (down) as changes to the outer nuclear and photoreceptor layers.

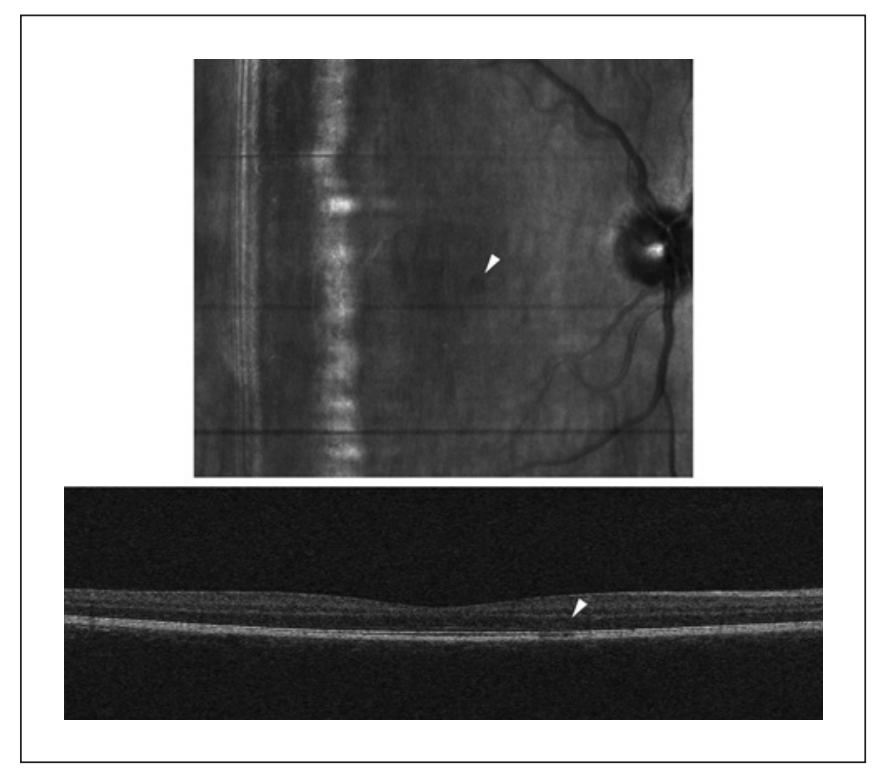

\section{Literature Analysis}

In November 2021, a search in the PubMed database was performed for "SARS-CoV-2," "COVID-19 vaccines," and "Acute Macular Retinopathy." To broaden the search, the references of all found articles were screened for additional sources. In total, thirteen papers of interest were retrieved [13-25]. All but 1 paper were case reports or case series; 1 article was a retrospective study [13]. Relevant data of all independent cases, such as number of reported cases, age, sex, COVID-19 and vaccination status, onset of complaints, and usage of oral contraceptives, were noted and analyzed using Microsoft Excel for Mac (version 16.54) in combination with the Analysis ToolPak add-in. Alpha was set at 0.05. Altogether, fourteen cases were found and split up in two separate groups. Seven cases were linked to a COVID-19 infection (Table 1), and the other 7 cases were linked to a vaccine against COVID-19 (Table 2).

In the group of cases linked to a COVID-19 infection, patients were on average 21.3 years older compared to the group of patients with AMN after vaccination, with 2 patients being 70 and 71 years old $[14,15]$. This difference was found to be significant (two-tailed Student's $t$ test assuming unequal variances, $p=0.0239$ ). There was no significant difference between sexes in both groups $\left(\chi^{2}\right.$ test, $\left.p=0.2367\right)$. The most reported vaccine was Vaxzevria ${ }^{\circledR}$ (also reported as Covishield ${ }^{\mathrm{TM}}$, viral vector vaccine; Oxford-AstraZeneca, Cambridge, UK) with 6 cases; in 1 case, Comirnaty ${ }^{\circledR}$ (mRNA vaccine; Pfizer/BioNTech, New York City, NY, USA) was used; $83.3 \%$ of cases were reported after the first dose of the vaccine (out of 2 doses) [14, 16-20]. No other vaccines were reported. This paper was written before any "booster" vaccinations were given. There was a significant difference in the use of oral contraceptives by female patients in both groups ( $\chi^{2}$ test, $p=0.0047$ ); however, it should be noted that this difference is because the papers in the COVID-19 group did not mention if patients did not use oral contraceptives. Average onset of complaints of AMN after COVID-19 was significantly later than AMN after vaccination (two-tailed Student's $t$ test assuming unequal variances, $p=0.0301$ ). In 2 cases, patients were diagnosed with AMN 9 and 4 days, respectively, before the first symptoms of COVID-19 [21, 22].

Data may be skewed due to the fact that the diagnosis of 1 reported case of AMN after COVID-19 might be disputed because the retinal image in this patient was different from all other cases, including intraretinal hemorrhages, Roth's spots, and subretinal fluid, alongside the typical OCT presentation of AMN [23]. In the case of Zamani et al. [22], there were also

\section{Karger'}


Table 1. Cases of AMN linked to COVID-19 infection

\begin{tabular}{lllll}
\hline Article & Sex & Age, years & Oral contraceptives & Timing \\
\hline Aidar et al. [15] & Female & 71 & Not applicable & 14 days after \\
Preti et al. [21] & Male & 70 & Not applicable & 4 days before \\
Zamani et al. [22] & Female & 35 & Not mentioned & 9 days before \\
Gascon et al. [23] & Male & 53 & Not applicable & 8 days after \\
Virgo et al. [24] & Female & 37 & Not mentioned & 35 days after \\
& Male & 32 & Not applicable & 16 days after \\
Giacuzzo et al. [25] & Female & 23 & Not mentioned & 14 days after \\
Total (average) & 57.1\% female & 45.9 (SD 19.1) & No data & 17.4 (SD 10.3) \\
\hline
\end{tabular}

Timing in is the amount of days AMN was diagnosed before or after the diagnosis of COVID-19. In the average, only the days after the diagnosis of COVID-19 were included.

SD, standard deviation.

Table 2. Cases of AMN linked to COVID-19 vaccines

\begin{tabular}{llllll}
\hline Article & Sex & Age, years & Oral contraceptives & Timing & Type of vaccine \\
Drüke et al. [14] & Female & 23 & Yes & 1 day & Vaxzevria (n/a) \\
Mambretti et al. [16] & Female & 22 & Yes & 2 days & Vaxzevria (1st) \\
& Female & 24 & Yes & 2 days & Vaxzevria (1st) \\
Book et al. [17] & Female & 21 & Yes & 3 days & Vaxzevria (1st) \\
Vinzamuri et al. [18] & Male & 34 & Not applicable & n/a & Vaxzevria (2nd) \\
Chen et al. [19] & Female & 21 & Yes & 2 days & Comirnaty (1st) \\
Bøhler et al. [20] & Female & 27 & Yes & 1 day & Vaxzevria (1st) \\
Total (average) & $85.7 \%$ & 24.6 & $100 \%$ of all & 2.2 days & \\
& female & (SD 4.5) & female patients & (SD 0.75) & \\
\hline
\end{tabular}

Timing in is the amount of days AMN was diagnosed after COVID-19 vaccination.

$\mathrm{n} / \mathrm{a}$, data not available; SD, standard deviation.

Roth's spots, but these were attributed to acute myeloid leukemia, for which the patient underwent chemotherapy.

Finally, 1 retrospective study was found, in which a similar rise of patients with AMN was seen. Azar et al. [13] analyzed all cases in a Paris hospital in France from 2019 to 2020 and found a significant rise in patients with AMN; one in 2019 and eleven in 2020. Four patients had tested positive for COVID-19. At the time of this study, COVID-19 vaccines were not yet available to the general public in France. Not all cases could be linked to COVID-19, but the rise of cases during the pandemic is striking.

\section{Conclusion and Discussion}

Viral infections and vaccines are already known to cause AMN, but the scale of the current COVID-19 pandemic and the rapid roll-out of vaccination programs worldwide have caused a surge of new cases, as seen in the above cases and the literature. It is hard to tell whether 
AMN occurs more often after COVID-19 or its vaccines, or that the sheer rise in numbers has caused this surge. Familiarity with the disease will benefit the diagnostic process in the ophthalmologist's office.

It has been theorized that an inflammatory reaction of a virus or vaccines trigger some vascular or thromboembolic event, causing ischemia of the deep retinal capillary plexus causing semipermanent relative scotomas. It is thought that the use of oral contraceptives predisposes for such an event, and hence AMN is seen more in young females [4, $16,18]$. The cases reported in this paper, and the analyzed reports from other countries, support this theory. The precise mechanism, however, remains unknown.

Interestingly, AMN that has been triggered by a COVID-19 infection can happen significantly later and at an older age than those triggered by a vaccine against COVID-19. Especially, the older age is a deviation from the typical characteristics of AMN. Therefore, AMN should be part of the differential for patients with sudden scotomas of all ages, especially during the ongoing pandemic. Mind that AMN has also been reported preceding the first symptoms of COVID-19 and could thus be a rare presenting symptom.

On average, in the available literature, symptoms of AMN arise 2.2 days after first vaccination; the 2 cases in this paper arose after 1.5 months after second vaccination. This could be explained by the fact that some patients notice their scotoma by coincidence, as was the case with patient A. Also, these patients have received a different vaccine (Spikevax; Moderna) than those reported in the literature so far, making this the first reported cases of AMN after this type of vaccine. It appears that use of oral contraceptives is a predisposing condition for AMN after vaccination against COVID-19, but this difference could also be explained by the age difference (and thus demand for oral contraceptives) between the groups.

To date, this is the largest case series of patients with AMN linked to COVID-19 or its vaccines. Prior to this paper, there have been more than a dozen similar reports during the period of 1 year. The recent rise of cases of AMN linked to the COVID-19 pandemic seems to confirm a suspected correlation; however, it should be noted that correlation does not imply causation, and that further research should be warranted. Due to the rarity of this disease, this could prove a difficult task.

\section{Statement of Ethics}

For solely reporting of cases, ethical approval was not required in accordance with local/ national guidelines. Written informed consent was obtained from 5 patients for publication of their relevant medical data and any accompanying images. One case of AMN could not be linked to COVID-19 and was therefore not reported in this series.

\section{Conflict of Interest Statement}

The authors declare no conflicts of interest.

\section{Funding Sources}

No funding was required for the realization of this paper. 


\section{Author Contributions}

Both authors, Maarten B. Jalink and Inge H.G. Bronkhorst, have made a significant contribution to this paper. Both authors have seen and treated the patients that were mentioned in this report. Maarten B. Jalink drew the initial draft, which was expanded upon by Inge H.G. Bronkhorst.

\section{Data Availability Statement}

All data generated or analyzed during this study are included in this article. Further enquiries can be directed to the corresponding author.

\section{References}

1 Bos PJ, Deutman AF. Acute macular neuroretinopathy. Am J Ophthalmol. 1975 Oct;80(4):573-84.

2 Bhavsar KV, Lin S, Rahimy E, Joseph A, Freund KB, Sarraf D, et al. Acute macular neuroretinopathy: a comprehensive review of the literature. Surv Ophthalmol. Sep-Oct 2016;61(5):538-65.

3 Turbeville SD, Cowan LD, Gass JD. Acute macular neuroretinopathy: a review of the literature. Surv Ophthalmol. 2003;48(1):1-11.

4 Fawzi AA, Pappuru RR, Sarraf D, Le PP, McCannel CA, Sobrin L, et al. Acute macular neuroretinopathy: longterm insights revealed by multimodal imaging. Retina. 2012 Sep;32(8):1500-13.

5 Liu JC, Nesper PL, Fawzi AA, Gill MK. Acute macular neuroretinopathy associated with influenza vaccination with decreased flow at the deep capillary plexus on OCT angiography. Am J Ophthalmol Case Rep. 2018;10: $96-100$.

6 Shah P, Zaveri JS, Haddock LJ. Acute macular neuroretinopathy following the administration of an influenza vaccination. Ophthalmic Surg Lasers Imaging Retina. 2018;49(10):e165-8.

7 Orphanet. Acute macular neuroretinopathy. Available from: https://www.orpha.net/consor/cgi-bin/OC_Exp. php?lng=EN\&Expert=488239 Accessed 2021 Nov 5.

8 Stoye E. China coronavirus: how many papers have been published? Nature. 2020 Jan 30. Online ahead of print.

9 COVID research: a year of scientific milestones. Nature. 2021 May 5. Online ahead of print.

10 Oran DP, Topol EJ. The proportion of SARS-CoV-2 infections that are asymptomatic: a systematic review. Ann Intern Med. 2021 May;174(5):655-62.

11 Nasiri N, Sharifi H, Bazrafshan A, Noori A, Karamouzian M, Sharifi A. Ocular manifestations of COVID-19: a systematic review and meta-analysis. J Ophthalmic Vis Res. 2021 Jan-Mar;16(1):103-12.

12 Rijksinstituut voor Volksgezondheid en Milieu (RIVM), the Dutch National Institute for Public Health and the Environment. Cijfers COVID-19 vaccinatieprogramma. Available from: https://www.rivm.nl/covid-19-vaccinatie/cijfers-vaccinatieprogramma Accessed 2021 Nov 16.

13 Azar G, Bonnin S, Vasseur V, Faure C, Salviat F, Clermont CV, et al. Did the CoVID-19 pandemic increase the incidence of acute macular neuroretinopathy? J Clin Med. 2021;10(21):5038.

14 Drüke D, Pleyer U, Hoerauf H, Feltgen N, Bemme S. Acute macular neuroretinopathy (AMN) following COVID-19 vaccination. Am J Ophthalmol Case Rep. 2021 Dec;24:101207.

15 Aidar MN, Gomes TM, de Almeida MZH, de Andrade EP, Serracarbassa PD. Low visual acuity due to acute macular neuroretinopathy associated with COVID-19: a case report. Am J Case Rep. 2021 Apr 30;22:e931169.

16 Mambretti M, Huemer J, Torregrossa G, Ullrich M, Findl O, Casalino G. Acute macular neuroretinopathy following coronavirus disease 2019 vaccination. Ocul Immunol Inflamm. 2021 May 19;29(4):730-3.

17 Book BAJ, Schmidt B, Foerster AMH. Bilateral acute macular neuroretinopathy after vaccination against SARSCoV-2. JAMA Ophthalmol. 2021 Jul 1;139(7):e212471.

18 Vinzamuri S, Pradeep TG, Kotian R. Bilateral paracentral acute middle maculopathy and acute macular neuroretinopathy following COVID-19 vaccination. Indian J Ophthalmol. 2021 Oct;69(10):2862-4.

19 Chen S, Hodge C. Comment on: "acute macular neuroretinopathy following COVID-19 vaccination". Eye. 2021 Sep 28:1-2. Online ahead of print.

20 Bøhler AD, Strøm ME, Sandvig KU, Moe MC, Jørstad ØK. Acute macular neuroretinopathy following COVID-19 vaccination. Eye. 2021 Jun 22:1-2. Online ahead of print.

21 Preti RC, Zacharias LC, Cunha LP, Monteiro MLR. Acute macular neuroretinopathy as the presenting manifestation of COVID-19 infection. Retin Cases Brief Rep. 2021 May 12.

22 Zamani G, Ataei Azimi S, Aminizadeh A, Shams Abadi E, Kamandi M, Mortazi H, et al. Acute macular neuroretinopathy in a patient with acute myeloid leukemia and deceased by COVID-19: a case report. J Ophthalmic Inflamm Infect. 2021 Jan 8;10(1):39. 
23 Gascon P, Briantais A, Bertrand E, Ramtohul P, Comet A, Beylerian M, et al. Covid-19-associated retinopathy: a case report. Ocul Immunol Inflamm. 2020 Nov 16;28(8):1293-7.

24 Virgo J, Mohamed M. Paracentral acute middle maculopathy and acute macular neuroretinopathy following SARS-CoV-2 infection. Eye. 2020;34(12):2352-3.

25 Giacuzzo C, Eandi CM, Kawasaki A. Bilateral acute macular neuroretinopathy following COVID-19 infection. Acta Ophthalmol. 2021 May 26. 